\title{
Cirurgia PIEZELÉTRICA ou PIEZOCIRURGIA em Odontologia: o sonho de todo cirurgião...
}

\author{
Maria Fernanda M-O Consolaro*, Eduardo Sant'Ana**, \\ Gastão Moura Neto***
}

Ao caminhar pela praia um cirurgião encontrou uma lâmpada mágica. Ao esfregá-la apareceu o gênio da lâmpada e prometeu conceder-lhe um desejo. Depois de muito pensar, o cirurgião formulou bem e proferiu seu pedido: desejo que produza uma serra cirúrgica que não lese tecidos moles, corte apenas o osso com a suavidade de quem corta um fragmento de queijo, sem esquentá-lo, com o máximo de precisão, sem sangramento e sem provocar muito edema. O gênio suspira aliviado e the responde: "Pois bem meu caro, seu desejo será facilmente atendido, pois sua serra foi inventada e já existe no mercado!".

Parece conto-de-fadas, mas trata-se de um sistema chamado Piezosurgery ${ }^{\circledR}$, desenvolvido pelo Dr. Tomaso Vercellotti em conjunto com os engenheiros da Mectron Medical Technology, Carasco, Genova, Itália, em 2007. Desde então, diversos estudos vêm testando e comprovando os benefícios desta nova tecnologia ${ }^{1-6,8-10}$. Dois tipos de aparelhos estão disponíveis, um médico e outro menor, odontológico.

O sistema adota um conceito já amplamente conhecido, o de piezeletricidade. Ao receber estímulos de pressão ou tensão, os cristais como o quartzo, por exemplo, são capazes de produzir

* Mestre e Doutora pela FOB-USP e ortodontista privada - Bauru / SP.

** Professor Livre Docente em Cirurgia e Traumatologia - FOB-USP e cirurgião privado.

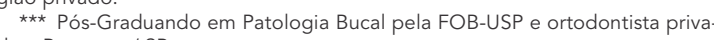
do - Botucatu / SP campos eletromagnéticos de intensidade exatamente iguais aos que receberam, muitas vezes de forma tão perfeita que são utilizados em relógios e em computadores, inclusive para marcar o tempo. Os cristais de hidroxiapatita apresentam a mesma propriedade piezelétrica, deformando-se em um campo elétrico. Esta tecnologia já é empregada na Odontologia, utilizando ondas de ultra-som para a remoção de cálculos dentários, por exemplo. Assim esta nova "serra" utiliza a capacidade de piezeletricidade óssea para desagregar os cristais de hidroxiapatita em um determinado plano, "cortando" o osso por meio de vibrações de ultra-som.

Este dispositivo piezelétrico consiste em uma plataforma que converte a corrente elétrica em ondas ultra-sônicas, por meio de um transdutor especial, ligado a uma peça de mão, anexa a bisturis ou pontas de corte, diamantadas ou de titânio, disponíveis em variadas formas (Fig. 1, 2). O ultra-som piezelétrico promove um padrão vibratório linear com freqüência de 24,7 a $29,5 \mathrm{kHz}$, com uma opção digital de modo reforçado "boosted" até $30 \mathrm{kHz}$, com oscilação de 60 a $210 \mu \mathrm{m}$ e uma potência que pode variar entre 2,8 a 16W, de acordo com a densidade do osso que se pretende cortar. A piezeletricidade é três vezes mais potente que ultra-sons comuns e, portanto, pode cortar tecidos altamente mineralizados, inclusive tecidos dentários duros.

A principal vantagem da cirurgia piezelétrica é que, uma vez em contato com tecidos moles, o 


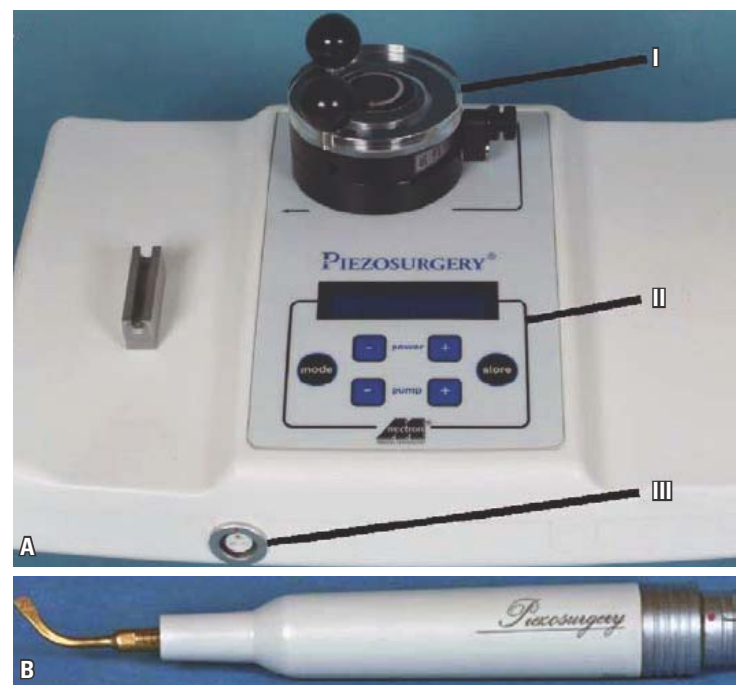

FIGURA 1 - Em A, o Sistema Mectron Piezosurgery®: (I) plataforma principal e painel digital, (II) bomba peristáltica, (III) saída para o conector da peça de mão. Em B, a peça de mão com uma ponta de corte inserida (extraído de EGGERS et al.').
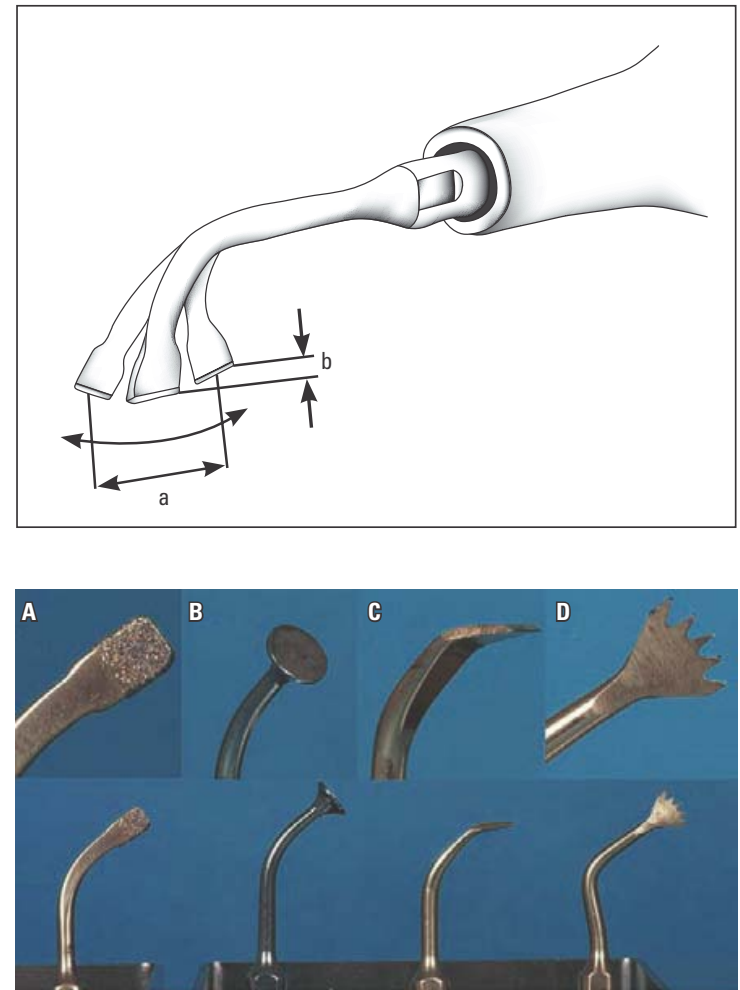

FIGURA 2 - Desenho da simulação do corte piezelétrico micrométrico longitudinal e série de pontas de corte disponíveis: (A) escalpo plano com ponta diamantada revestida superficialmente por nitrido de titânio; (B) compressor cônico, arredondado, horizontal; (C) removedor de osso, revestido por nitrido de titânio e (D) serra afiada, revestida de nitrido de titânio (extraído de ROBIONY et al. ${ }^{4}$ e EGGERS et al. $\left.{ }^{1}\right)$. dispositivo ativo de corte cessa sua atividade, preservando totalmente a integridade de vasos e nervos, freqüentemente e/ou acidentalmente comprometidos ao usar serras cirúrgicas convencionais ou brocas.

Ao utilizar serras comuns ou brocas em osteotomias, há necessidade de se aplicar pressão, mesmo que discreta, para que se obtenha o corte, implicando em certo grau de aquecimento, tanto do osso quanto dos tecidos moles adjacentes. $\mathrm{O}$ corte piezelétrico não deve utilizar pressão, pois o excesso de força sobre o instrumento interrompe a sua atividade sobre o osso. Deve-se realizar apenas a apreensão firme da microserra durante o corte, o que resulta em um mínimo aquecimento, diminuindo o risco de osteonecrose, garantindo a vitalidade dos osteócitos. Quanto mais suave a pressão
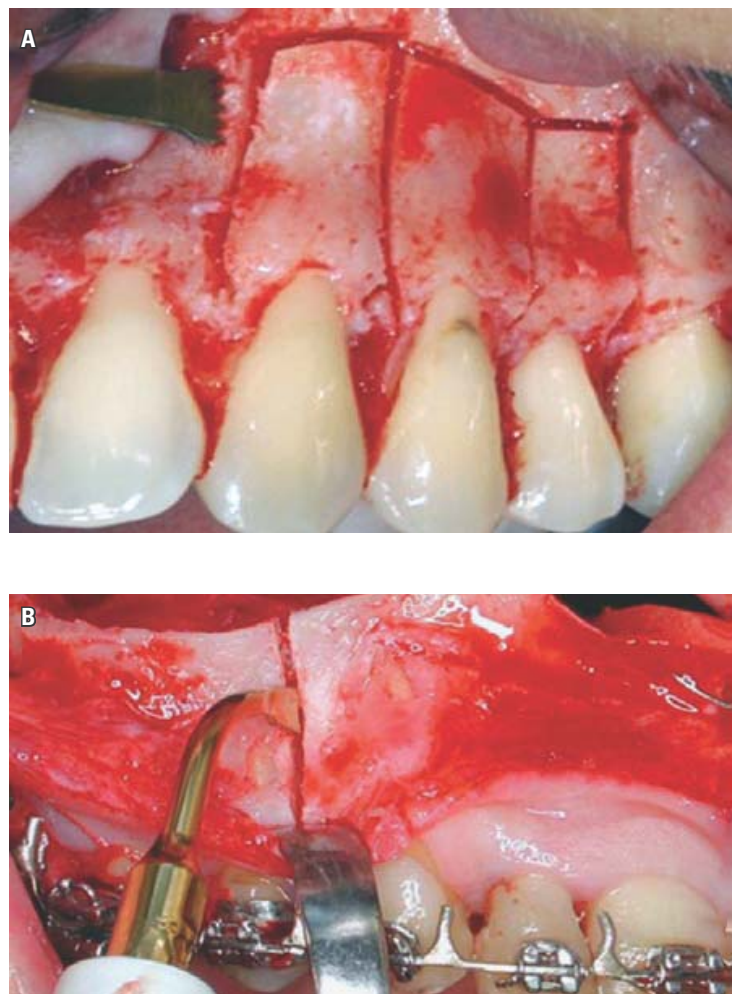

FIGURA 3 - Osteotomias vertic ais, estreitas e precisas realizadas com a serra piezelétrica entre as raízes dentárias. (A) Corticotomias microcirurgicas ao redor dos dentes; (B) osteotomias para multisegmentação de maxila com mínimo descolamento mucogengival (Piezosurgery ${ }^{\circledast}$ slide presentation e ROBIONY et al. $\left.{ }^{4}\right)$. 
da serra piezelétrica sobre o osso, mais linear a vibração do instrumento e melhor o corte.

O sistema Piezosurgery ${ }^{\circledR}$ apresenta também uma bomba peristáltica de solução salina $(0,9 \%)$ ou soro fisiológico estéril, mantida em uma unidade a $4^{\circ} \mathrm{C}$. A solução é expelida por meio de um jato anexo à ponta ativa, em um fluxo que pode ser ajustado entre 0 a $60 \mathrm{ml} / \mathrm{min}$, refrigerando a superfície óssea e eliminando detritos da área de corte.

Uma vez que não lesa tecidos moles, o dispositivo reduz sensivelmente o sangramento durante as intervenções cirúrgicas, melhorando a visibilidade durante o procedimento e diminuindo fenômenos inflamatórios indesejáveis, como edema e dor.

O aparelho corta o osso seletivamente, com suavidade, precisão micrométrica e com qualidade comprovadamente superior à das serras oscilatórias convencionais - que utilizam macrovibrações ou brocas cirúrgicas - utilizando macrorotações ${ }^{2}$, preservando tecidos moles e feixes vasculonervosos. A análise microscópica de tecidos ósseos obtidos durante procedimentos cirúrgicos com a microserra piezelétrica revelou integridade celular nas superfícies ósseas e ausência de necrose por coagulação, além de preservação de vitalidade pulpar em cirurgias odontológicas $4,5,8$.

Os primeiros trabalhos odontológicos a respeito descreveram a utilização da serra piezelétrica em cirurgias pré-protéticas e enxertos sinusais, depois as técnicas foram se diversificando para procedimentos como: osteotomias, osteoplastias, levantamentos de seio, expansão de rebordo alveolar, extração de raízes com anquilose alveolodentária, corticotomias de precisão para movimentação dentária, osteotomias de segmentação de maxila e para expansão rápida cirurgicamente assistida, distração osteogênica, obtenção de osso autógeno para enxertos, entre outros.

A "Ortodontia cirúrgica" como é abordada no www.piezosurgery.com será imensamente beneficiada pelo novo dispositivo de corte. A técnica de deslocamento dentário monocortical e distração ligamentar ou monocortical tooth dislocation and ligament distraction (MTDLD) consiste na movimentação ortodôntica convencional associada a corticotomias microcirúrgicas ao redor dos dentes, eliminando a resistência da cortical, diminuindo o tempo de tratamento ortodôntico em até $60 \%$ a $70 \%$. Com a microserra piezelétrica estas corticotomias podem ser realizadas com precisão micrométrica ${ }^{9}$ (Fig. 3A).

A expansão rápida da maxila assistida cirurgicamente (ERMAC), procedimento já bastante popularizado no meio ortodôntico, pode ser realizada tanto sob anestesia geral quanto local, mas com diferenças quanto à técnica cirúrgica. Com freqüência, a preferência do paciente é a realização da cirurgia sob anestesia local. A disjunção da união pterigomaxilar entre a placa pterigóide do osso esfenóide e a porção posterior da maxila é altamente contra-indicada nas cirurgias sob anestesia local, pela dificuldade de visualização da artéria palatina maior, que pode resultar em profuso sangramento de difícil controle, já que não é possível realizar uma osteotomia completa de maxila sob anestesia local. A impossibilidade de realização da separação pterigomaxilar, muitas vezes, impede uma expansão regular das maxilas, que por vezes acaba abrindo mais na região anterior que posterior. A disjunção pterigomaxilar seria a única garantia de um padrão previsível de expansão maxilar ${ }^{4,5}$.

Os trabalhos sobre ERMAC e piezocirurgia revelam que a utilização de vibração ultra-sônica permite a realização desta disjunção pterigomaxilar completa, garantindo uma expansão regular e maior, principalmente quando há necessidade de grande quantidade de expansão posterior. $\mathrm{O}$ sangramento trans e pós-cirúrgico é muito reduzido, uma vez que a microserra piezelétrica preserva totalmente a artéria palatina maior e outras estruturas anatômicas, sem alteração de temperatura e mínima ocorrência de edema ou equimoses pósoperatórias, surpreendendo os pacientes ${ }^{4,5}$.

As cirurgias ortognáticas envolvendo Le Fort I com multisegmentação de maxila são consideradas de alto risco de complicações para tecidos moles e 
duros. As osteotomias são geralmente realizadas nas proximidades de estruturas anatômicas delicadas, como os tecidos moles vestibular e lingual do palato, que provêm a vascularização óssea, por meio do periósteo. Os retalhos mucogengivais ou palatinos podem ser danificados durante as osteotomias, principalmente as verticais e na linha mediana, com comprometimento da vascularização óssea, dentária e periodontal. A utilização de serras oscilatórias e brocas rotatórias, freqüentemente, promove aquecimento, podendo ocasionar osteonecrose marginal e comprometimento do reparo ósseo. Do mesmo modo, a vascularização dos retalhos pediculados de tecido mole pode ser prejudicada direta ou indiretamente pelo aquecimento, assim como os tecidos periodontais adjacentes, com perda de osso alveolar interdentário, retração ou atrofia gengival e lesões no cemento radicular. Diversos estudos indicam que os instrumentos de corte convencionais podem comprometer, nos mais diversos graus, a vascularização e a vitalidade pulpar dos dentes envolvidos em segmentações de maxila ${ }^{3}$.

Por meio de vibrações lineares, o sistema Piezosurgery ${ }^{\circledR}$ possibilita a obtenção de cortes com precisão e seletividade, com um mínimo de dano aos tecidos moles e duros, tanto nas porções vestibulares quanto palatinas da maxila. Os corte micrométricos permitem a realização de osteotomias entre as raízes dentárias. A facilidade de manuseio do instrumento, irrigação apropriada e sangramento reduzido possibilitam uma melhor visibilidade da área de corte, evitando a necessidade da utilização "cega" de brocas e cinzéis ${ }^{3}$ (Fig. 3B).

As osteotomias paramedianas são recomendadas porque o osso palatino da linha média é espesso e a mucosa palatina muito fina, de modo que as osteotomias medianas são consideradas de alto risco de necrose dos tecidos moles do palato. As evidências recentes revelam que as osteotomias medianas realizadas com este sistema podem ser executadas com um risco mínimo de lesão ou necrose da mucosa palatina ${ }^{3}$.

As perspectivas de utilização deste instrumento prometem revolucionar importantes paradigmas nas diversas áreas da Cirurgia. É o fruto de uma idéia ousada de um cirurgião que se aventurou em imaginar a serra dos seus sonhos; encontrando em um conceito conhecido, uma aplicação totalmente inovadora. Trata-se de um clínico, formado em Medicina e Cirurgia, especialista em Odonto-Estomatologia, dedicado ao consultório privado, atuando como periodontista e a implantodontista. Como professor, atua na qualidade de visitante em algumas universidades, nem por isso deixa de ser um exemplo para pesquisadores e cientistas de todo o mundo?

\section{REFERÊNCIAS}

1. EGGERS, G.; KLEIN, J.; BLANK, J.; HASSFELD, S. Piezosurgery: an ultrasound device for cutting bone and its use and limitations in maxillofacial surgery. Br. J. Oral Maxillofac. Surg., Edinburgh, v. 42, no. 5, p. 451-453, Oct. 2004.

2. GLEIZAL, A.; BERA, J. C.; LAVANDIER, B.; BEZIAT, J. L. Piezoelectric osteotomy: a new technique for bone surgeryadvantages in craniofacial surgery. Childs Nerv. Syst., Berlin v. 23, no. 5, p. 509-513, May 2007.

3. ROBIONY, M.; POLINI, F.; COSTA, F.; VERCELLOTTI, T.; POLITI, M. Piezoelectric bone cutting in multipiece maxillary osteotomies. J. Oral Maxillofac. Surg., Philadelphia, v. 62, no. 6, p. 759-761, June 2004

4. ROBIONY, M.; POLINI, F.; COSTA, F.; ZERMAN, N.; POLITI, M. Ultrasonic bone cutting for surgically assisted rapid maxillary expansion (SARME) under local anaesthesia. J. Oral Maxillofac. Surg., Philadelphia, v. 36, no. 3, p. 267-269, Mar. 2007.

5. ROBIONY, M.; POLINI, F.; COSTA, F.; ZERMAN, N.; POLITI, M. Ultrasound bone cutting for surgically assisted rapid maxillary expansion under local anesthesia. Preliminary results. Minerva Stomatol., Torino, v. 56, no. 6, p. 359-368, June 2007.

6. STÜBINGER, S.; KUTTENBERGER, J.; FILIPPI, A.; SADER, R.; ZEILHOFER, H. F. Intraoral piezosurgery: preliminary results of a new technique. J. Oral Maxillofac. Surg., Philadelphia, v. 63, no. 9, p. 1283-1287, Sept. 2005.

7. VERCELLOTTI, T. Piezoelectric surgery in Implantology: a case report - a new piezoelectric ridge expansion technique. Int. J. Periodontics Restorative Dent., Chicago, v. 20, no. 4, p. 358-365, Aug. 2000

8. VERCELLOTTI, T. Technological characteristics and clinical indications of piezoelectric bone surgery. Minerva Stomatol. Torino, v. 53, no. 5, p. 207-214, May 2004

9. VERCELLOTTI, T.; PODESTA, A. Orthodontic microsurgery: a new surgically guided technique for dental movement. Int. J. Periodontics Restorative Dent., Chicago, v. 27, no. 4, p. 325-331, Aug. 2007.

10. VERCELLOTTI, T.; DELLEPIANE, M.; MORA, R.; SALAMI, A. Piezoelectric bone surgery in otosclerosis. Acta Otolaryngol., Stockholm, v. 127, no. 9, p. 932-937, Sept. 2007 